\title{
Prevalência e determinantes de obesidade e sobrepeso em mulheres em idade reprodutiva residentes na região semiárida do Brasil
}

\author{
Prevalence and determinants of obesity and overweight among \\ reproductive age women living in the semi-arid region of Brazil
}

\author{
Luciano Lima Correia $^{1}$ \\ Dirlene M afalda Ildefonso da Silveira ${ }^{2}$ \\ Anamaria Cavalcante e Silva ${ }^{3}$ \\ JocileideSales Campos ${ }^{3}$ \\ Márcia M aria Tavares $M$ achado ${ }^{1}$ \\ Hermano Alexandre Lima Rocha ${ }^{1}$ \\ Antônio José Ledo Alves da Cunha ${ }^{4}$ \\ Ana Cristina Lindsay ${ }^{5}$
}

${ }^{1}$ Departamento deSaúde Comunitária, Faculdadede M edicina, Universidade Federal do Ceará. Rua Professor Costa M endes $1.608,50$ andar, Campus Rodolfo Teófilo.

60414-130 Fortaleza CE. correialuciano@hotmail.com ${ }^{2} \mathrm{~N}$ úcleo de Epidemiologia, Secretaria de Saúde do Estado do Ceará.

${ }^{3}$ Faculdade de M edicina Christus, Fortaleza, Ceará. ${ }^{4}$ Departamento de Pediatria, Universidade Federal do Rio de Janeiro. ${ }^{5}$ Department of Nutrition, $\mathrm{H}$ arvard School of Public Health.
Abstract Thestudy estimated the prevalenceand determinants of overweight and obesity in women of reproductive age in the Semi-Arid Region of Brazil. In the cluster sampling of 8,000 households of the state of Ceará, 6,845 women were surveyed. Overweight and obesity weremeasured by the body mass index and risk factors identified by multivariate analysis. The prevalences of overweight and obesity were $32.6 \%$ and $16.1 \%$, respectively. After adjusted analysis, obesity was positively associated with age $>30$ years ( $P R=1.55)$, marital status, married ( $P R=1.36)$, elementary education $(P R=1.40)$, age at menarche $<12$ years $(O R$ $=1.59)$, having $>1$ child $(P R=1.65)$ and contraceptive use $(P R=1.31)$. Specific health and nutritional conditions were identified as highly associated to morbid obesity: hypertension ( $P R=3.11$ ), diabetes ( $P R=2.08)$, dissatisfaction with body image ( $P R=4.26)$ and procedures for weight loss $(P R=2.73)$. The study concluded that overweight and obesity are highly prevalent in the Semi-Arid Region of Brazil. Education was the only socioeconomic determinant considered as amenable to change. The reproductive risk factors identified enforce the need to mobilize the services of prenatal and postpartum care and family planning to prevent and control obesity. Key words O besity, O verweight, Women'shealth, Nutritional transition in Brazil, Epidemiology
Resumo 0 estudo estimou a prevalência e identificou fatores determinantes do sobrepeso e obesidade em mulheres em idade fértil na região semiárida do Brasil. $\mathrm{Na}$ amostra por conglomerados de oito mil domicílios do estado do Ceará, foram pesquisadas 6.845 mulheres. Sobrepeso e obesidade foram medidos pelo Índice de M assa Corporal, utilizando-se a análise multivariada para a identificação dos fatores determinantes. As prevalências de sobrepeso e obesidade foram de $32,6 \%$ e $16,1 \%$, respectivamente. Na análiseajustada, a obesidade esteve positivamente associada a: idade $>30$ anos ( $R P=1,55)$, estado civil casada $(R P=1,36)$, escolaridadefundamental $(R P=1,40)$, idade na menarca $<12$ anos ( $R P=1,59)$, ter tido $>1$ filho $(R P=1,65)$, uso de contraceptivos $(\mathrm{RP}=1,31)$. Os fatores relacionados à saúde e à nutrição, referentes à obesidade mórbida, foram: hipertensão arterial $(\mathrm{RP}=3,11)$, diabetes $(R P=2,08)$, insatisfação com a imagem corporal $(R P=4,26)$ e procedimentos para perder peso $(R P=2,73)$. Sobrepeso e obesi dade são altamente prevalentes na região semiárida. A educação foi 0 único fator socioeconômico passível de ser modificado. O sfatores reprodutivosidentificadosapontam para a necessi dade de mobilização dos serviços de pré natal, pós-parto e planejamento familiar na prevenção e no controle da obesidade. Palavras-chave O besidade, Sobrepeso, Saúde da mulher, Transição nutricional, Epidemiologia 
Introdução

As crescentes taxas de prevalência de sobrepeso e obesidade, correntemente registradas no mundo desenvolvido eem desenvolvimento, tem categorizado esta condição como um grave problema de saúde públical-6 já sendo reconhecido pela Organização Mundial da Saúde (OMS) como uma epidemia global ${ }^{7}$. N os Estados Unidos, um terço da população adulta é considerada obesa ${ }^{8}$, sendo que estimativas globais apontam para um bilhão de indivíduos com excesso de peso e 315 milhões com obesidade?.

Sobrepeso e obesidade representam uma sé ria ameaçaà saúde do adulto, estan do fortemente associadas a um risco aumentado de doenças crônicas degenerativas, particularmente doenças cardiovasculares, diabetes mellitus tipo II e outros distúrbios endócrinos e metabólicos, osteoartrite e certos tipos de câncer, ${ }^{5,7,10-13}$. Além da doença orgânica, o problema do peso excessivo traz prejuízos para a qualidade de vida, com a limitação da prática de atividades físicas ${ }^{14,15}$, e para a saúde mental, favorecendo a insatisfação com a imagem corporal que, por sua vez, implica redução da autoestima ${ }^{16,17}$. 0 problema do peso excessivo, acompanhado de todas as suas consequências, torna-seainda mais preocupantequando se constata que elevadas proporções da população são afetadas e em faixas etárias cada vez mais joven $\varsigma^{6,18}$.

$\mathrm{N}$ a região semiárida do país, a obesidade como problema de saúde pública é um fenômeno bastanterecente, uma vez que esta região sempre se caracterizou pelo pobre perfil nutricional de sua população, consequente aos indicadores socioeconômicos e ambientais desfavoráveis, permanentemente registrados. Estudo realizado nesta região, por sua vez, observou que populações "adaptadas" a um estado crônico de fome podem apresentar uma suscetibilidade aumentada à obesidade ${ }^{19}$.

0 presente estudo analisa dados de um le vantamento populacional sobre saúde maternoinfantil realizado com oito mil famílias residentes no Ceará, um dos estados mais representativos do semiárido brasileiro, porquanto apresenta a totalidade de seu território encravado nesta região.

População-alvo deste levantamento, as muIheres em idade reprodutiva se constituem em um grupo ainda pouco estudado com relação a sua situação nutricional na região semiárida, embora indicadores regionais apontem para uma crescente el evação das taxas de morbimortal ida- deprecoce por doenças crônico-degenerativas na população feminina.

Mulheres com filhos, por sua vez, são naturalmente motivadas a participarem em programas preventivos, sendo um alvo preferencial em atividades que promovam a qualidade de vida eo controle de peso ${ }^{20}$. Estudos que meçam a magnitude do problema do sobrepeso e da obesidade neste grupo populacional, identificando os fatores associados, fazem-se necessários, contribuindo para um melhor direcionamento das políticas públicas voltadas para a sua prevenção e controle $e^{21}$.

D essa forma, este estudo teve como objetivos estimar a prevalência e identificar os fatores determinantes do sobrepeso e da obesidade em mulheres em idade fértil residentes na região semiárida do Brasil.

\section{Métodos}

O estudo de desenho transversal pesquisou uma amostra representativa da população de mulheres em idadereprodutiva, especificamentena faixa etária de 20 a 49 anos, residentes no estado do Ceará, Nordeste do Brasil. 0 Ceará caracterizase como um dos estados mais pobres do país, com a inclemência do clima semiárido, queabrange $95 \%$ do seu território, refletindo-se, marcadamente, em sua população de 8,2 milhões de habitantes. Seu desenvolvimento econômico (indústrias e turismo) se concentra na capital, Fortaleza com uma estimativa de 2,3 milhões de habitantes, e na região metropolitana. N os demais municípios do interior, que concentram dois terços da população, prevalecea agricultura de subsistência, que alterna anos de safra com períodos recorrentes de seca. Os benefícios previdenciários (aposentadorias) e governamentais (bolsas de auxílios públicos) oferecidos às famílias têm se tornado a fonte de renda mais estável da população do interior, substancialmente garantindo o funcionamento da economia desta região.

0 estudo aplicou a técnica de amostragem por conglomerados, utilizando setores censitários do Instituto Brasileiro de Geografia e Estatística (IBGE) eestratificação entrea capital, Fortal eza, e o interior do estado. Os setores censitários resultam da divisão de cada município em áreas geográficas de extensões variáveis, mas com população uniforme, em torno de trezentas famílias.

O tamanho da amostra, calculado em oito mil domicílios, representando cerca de $35 \mathrm{mil}$ pessoas e sete mil mulheres de 20 a 49 anos, foi inicialmente estabelecido no primeiro estudo da 
série, em 1986, calculado para a estimativa da taxa demortalidadeinfantil do estado, mas mantido nos demais estudos da série. 0 tamanho da amostra inicial foi multiplicado por um fator de correção igual a 2, contemplando o efeito de delineamento próprio das amostragens por conglomerado; além disso, foi acrescido em 10\% para compensar eventuais perdas.

Para garantir a representatividade da população estudada, a seleção dos municípios, setores edomicílios foi realizada deforma al eatória, obedecendo a um processo de amostragem de múltiplos estágios ${ }^{22}$, quais sejam:

(1) Sorteio de quarenta municípios, sendo 13 na região metropolitana de Fortal eza ( $1 / 3$ da população) e 27 na região do interior. A seleção dos municípios foi realizada de forma sistemática de acordo com o seu peso populacional, a partir da lista de todos os municípios com suas respectivas populações, sequenciados por região, para garantir uma adequada distribuição geográfica. A partir deste processo, um município de grande porte pode ser sorteado mais de uma vez, tendo o seu peso populacional respeitado na amostra. Assim, Fortaleza foi sorteada dez vezes, eos demais, uma vez cada. No final, 31 municípios compuseram a amostra, em vez de quarenta.

(2) Em cada município, dez setores censitários das zonas urbanas e rurais foram selecionados por sorteio, permitindo assim que todas as áreas, mesmo as mais remotas, pudessem ser representadas. $\mathrm{Na}$ capital, Fortal eza, selecionada dez vezes, cem setores cen sitários do I BGE foram aleatoriamente selecionados.

(3) Uma vez definido o setor e seu respectivo mapa obtido do IBGE, foi definida por sorteio a local ização do conglomerado de vinte casas a serem pesquisadas. Novamente de forma al eatória, foi determinado o ponto de partida do conglomerado, ou seja, o primeiro domicílio a ser visitado. A partir deste, vinte domicílios consecutivos foram visitados, seguindo-se regras específicas: (i) visitas às casas obedecendo ao sentido antihorário; (ii) não inclusão no conglomerado de vinte casas dos estabelecimentos comerciais e das casas sem moradores, sendo estas substituídas por outras vizinhas; (iii) no caso de famílias ausentes, até três retornos foram realizados na tentativa de obtenção dos dados do domicílio.

(4) Em cada domicílio, todas as mulheres na faixa etária da pesquisa nele residentes tiveram suas medidas antropométricas aferidas e os respectivos questionários aplicados.

Para a avaliação do estado nutricional das mulheres foi realizada a verificação de peso, esta- tura e circunferência abdominal. Estas medidas permitiram o cálculo do índice de massa corpórea (IMC), indicador querelaciona peso ealtura, de acordo com gênero eidadeespecíficos, segundo parâmetros recomendados pela $\mathrm{OM} \mathrm{S}^{7}$. Os distúrbios nutricionais foram classificados de acordo com os parâmetros a seguir: obesidade de grau I: $30,0-34,9 \mathrm{~kg} / \mathrm{m}^{2}$; obesidade de grau II: $\geq 35,0 \mathrm{~kg} / \mathrm{m}^{2}$; sobrepeso: 25,0-29,9 kg/m²; eutrofia: $18,5-24,9 \mathrm{~kg} / \mathrm{m}^{2}$; desnutrição: < 18,5 kg/m².

Em cada domicílio foram obtidas as medidas antropométricas de todas as mulheres residentes, sendo em seguida realizadas as suas respectivas entrevistas, utilizando-se questionários específicos. 0 peso foi aferido por meio de balanças eletrônicas portáteis, tipo Tanita, com capacidade de $150 \mathrm{~kg}$ e calibragem de 0,1 kg, enquanto a estatura foi medida com antropômetros portáteis de 65 a $200 \mathrm{~cm}$, com precisão de 0,5 $\mathrm{cm}$. Os equipamentos foram calibrados regularmente, utilizando medidas padronizadas, no início do dia e a cada 25 mensurações.

A equipe técnica do estudo foi constituída de uma coordenação e de três equipes de pesquisadores de campo, compostas cada uma de um supervisor eoito antropometristas/entrevistadores, todos de nível superior, com formação na área de nutrição, saúde ou social. Os antropometristas trabalharam em duplas, cada uma cobrindo um setor (conglomerado de vinte casas) por dia.

As informações foram coletadas durante 0 período de julho a dezembro de 2007, utilizando-se dois questionários. 0 primeiro registrou as informações de cada domicílio incluído na amostra. 0 segundo questionário coletou informações de todas as mulheres de 20 a 49 anos residindo nos domicílios visitados.

Foi coletada uma vasta gama de informações de interesse sobre as mulheres e suas famílias, que incluíram: características biológicas (idade, situação conjugal etc.); composição da família (número e idade dos membros, parentesco etc.); dados socioeconômicos (educação, trabalho, renda familiar, suplementação de renda [bolsas] e alimentar etc.); características do domicílio (possessões domésticas, tipo de construção, número de compartimentos, água e saneamento, aglomeração etc.); padrão alimentar (hábitos alimentares, disponibilidade e consumo de alimentos, adoção de dietas, aleitamento materno etc.); padrão comportamental (tipo efrequência deatividades físicas, tipo detrabalho e delazer, percepção corporal etc.); padrão reprodutivo (paridade, número defilhosvivos, atenção prénatal etc.); histórico de saúde e morbidade da mu- 
Iher (utilização de serviços de saúde, antecedentes demorbidade, incluindo hipertensão arterial ediabetes); medidas antropométricas (peso, altura, circunferência abdominal).

Os questionários foram revisados diariamente pelos supervisores de campo para a identificação de erros de preenchimento e sua correção, nos casos em que isto era possível. Duranteo trabalho de campo, uma subamostra de mulheres foi repesada eteve sua estatura remedida pelos supervisores, em uma base cega, como forma de controle de qualidade do trabalho dos antropometristas.

Os dados foram digitados duplamente utilizando-se o programa Epilnfo (CDCWHO) e analisados estatisticamenteatravés do SPSS V14.0 for Windows.

Um modelo conceitual hierarquizado (Figura 1) mostra as várias inter-relações entre os diferentes fatores de risco. Os desfechos estudados foram o sobrepeso e a obesidade (desfechos primários) e um grupo de variáveis relacionadas à saúde, quais sejam, a hipertensão arterial eo diabetes mellitus, a nutrição, a satisfação com a imagem corporal e as ações visando à perda de peso (desfechos secundários).

A análise inicial consistiu da tabulação da distribuição de frequências de fatores sociodemográficos e de morbidade de acordo com o estado nutricional. N este estudo transversal, a razão de pre valência $(R P)$ foi estimada de modo similar à razão de incidência cumulativa em estudos de coorte. 0 testederazão de máxima verossimilhança com significância bicaudal foi usado para avaliar a força das associações. 0 modelo de regressão proporcional de Cox foi utilizado para investigar se a força da associação encontrada na análise univariada era significantemente afetada pela presença de eventuais variáveis confundidoras. Tem sido demonstrado que, assumindo um período constante derisco, o modelo de Cox podeser adaptado para estimar a RP em estudos transversai ${ }^{23,24}$, sendo arguído que, nestes estudos, esta medida produz melhores estimativas de risco do que a 0 dds Ratio ${ }^{25}$. $\mathrm{Na}$ análise multivariada foi utilizada a estratégia hierárquica ${ }^{26}$, na qual o processo de modelagem backwards incluiu, estrato por estrato, todas as variáveis que no modelo conceitual alcançaram o nível de significância de 5\%. Permaneceram no modelo final somente os fatores associados ao desfecho que no teste de razão de máxima verossimilhança apresentaram significância menor que $5 \%$.

0 fato denão ter sido utilizado fator de ponderação, aplicável em amostragens por conglomerado, implica que, eventualmente, algum determinante que estaria associado ao desfecho pode na análise não ter alcançado o nível de significância estabelecido. Entretanto, as estimativas apresentadas nos resultados que se mostram estatisticamente significativas estão corretas.

\section{Resultados}

Do total de 6.845 mulheres na faixa etária de 20 a 49 anos de idade quetiveram seu estado nutricional avaliado, 16,1\% apresentavam índice demassa corpórea (IMC) compatível com obesidade e $32,6 \%$ com o sobrepeso. Ao todo, $48,7 \%$ destas mulheres em idade reprodutiva e produtiva conviviam com níveis excessivos de peso (Tabela 1). Observou-se que este problema evoluiu com a idade, sendo quea prevalência da obesidade praticamente dobrou da faixa etária dos 20 para os 30 anos, passando de $11 \%$ para cerca de $20 \%$. A desnutrição, um distúrbio nutricional tradicionalmente associado à região semiárida, afetou somente $3,7 \%$ da população demulheres, sendo, ao contrário do excesso de peso, mais prevalente entre as mulheres mais jovens (5,7\%). As altas taxas de obesidade e sobrepeso e a baixa taxa de desnutrição refletem o corrente estado do processo de transição nutricional prevalente na região semiárida do Brasil, particularmente no que se refere ao grupo populacional estudado.

A seguir, são comparados os resultados encontrados para mulheres eutróficas e com obesidade. Quando ajustadas entre si, as variáveis idade, educação, situação conjugal, idade na menarca, número defilhostidoseutilização de métodos anticonceptivos foram encontradas como as meIhores preditoras da obesidade (Tabela 2). MuIheres nafaixa de 30 a 39 anos tiveram $55 \%$ a mais de risco de obesidade, em comparação às mulheresnafaixa imediatamenteinferior de 20 a 29 anos. Mulheres com nível educacional incipiente, ou seja, menos de cinco anos de estudos, tiveram um risco $40 \%$ maior de obesidade, em comparação àquela com nível superior. Não houve diferença significante no índice de obesidade entre mulheres com nível médio de educação ecom nível universitário. Mulheres que viviam com um companheiro (casadas ou em união conjugal) apresentaram um excesso de risco de obesidade de $36 \%$, em relação às mulheres que eventual menteconsideraram não possuir um companheiro, incluídas as solteiras, separadas e viúvas.

O início precoce da puberdade apresentou-se como um importantefator determinante da obesidade em mulheres que tiveram o primeiro episódio menstrual antes dos doze anos de idade, 
apresentando um risco 59\% maior de obesidade em comparação àquelas que apresentaram a menarca após a idade de doze anos. A reprodução em si também se manifestou como um fator determinante, com mulheresjá no primeiro filho apresentando um risco de obesidade $43 \%$ mais elevado do que as nulíparas. Um segundo filho tornou este risco ainda maior, aumentando-o para $65 \%$. 0 desejo de não engravidar, por sua vez, também se manifestou como um fator determinante, em mulheres quefaziam uso de mé todos contraceptivos, apresentando uma probabilidade 31\% maior de estarem obesas do que aquelas que não os usavam.

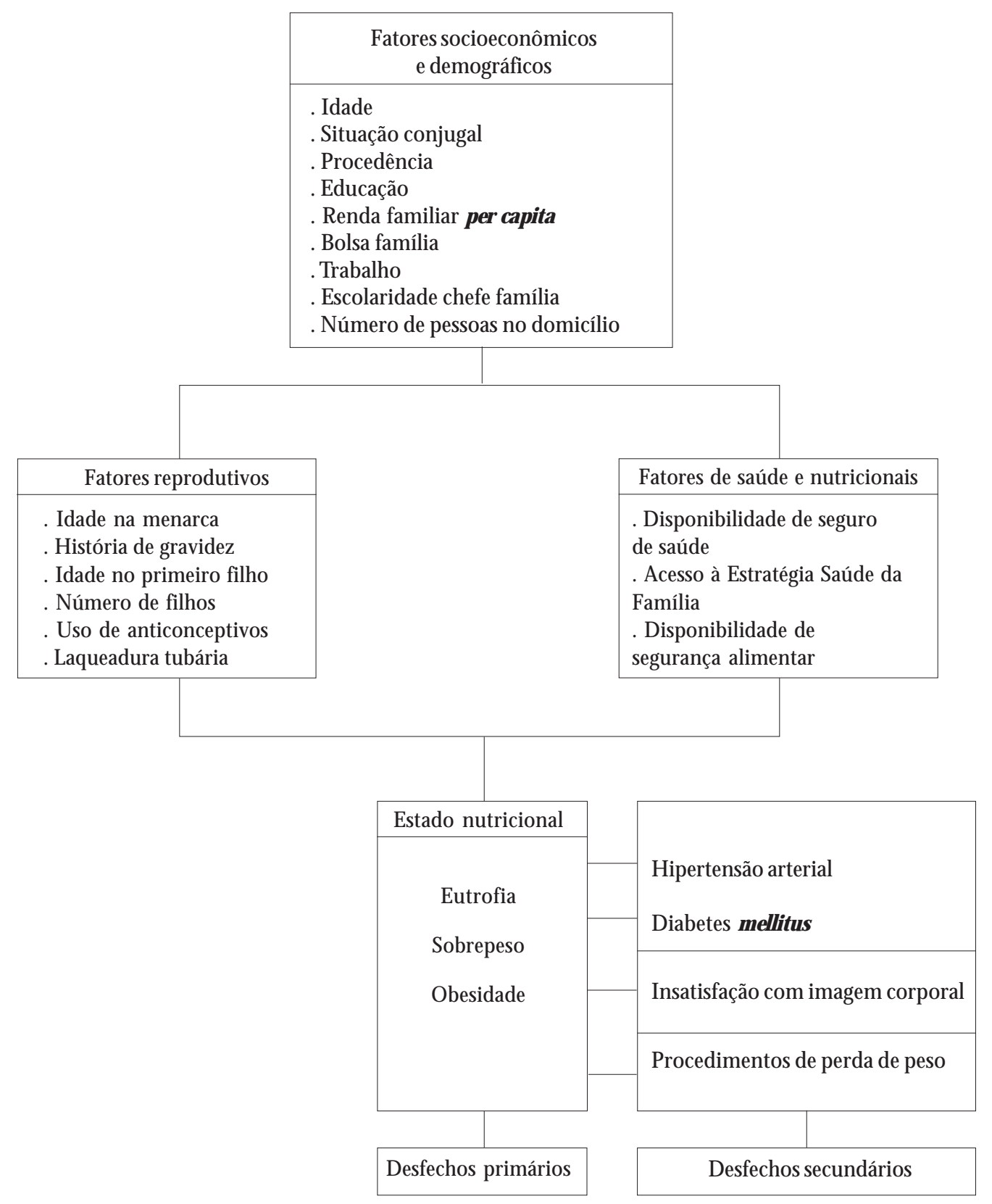

Figura 1. Esquema simplificado do modelo conceitual. 
Tabela 1. Distribuição percentual dos distúrbios nutricionais em mulheres adultas em idade reprodutiva. Ceará, Brasil, 2007.

\begin{tabular}{lcccc}
\hline Estado nutricional & $\begin{array}{c}\text { Desnutrição } \\
(\mathrm{n}=254)\end{array}$ & $\begin{array}{c}\text { Eutrofia } \\
(\mathrm{n}=3.252)\end{array}$ & $\begin{array}{c}\text { Sobrepeso } \\
(\mathrm{n}=2.234)\end{array}$ & $\begin{array}{c}\text { Obesidade } \\
(\mathrm{n}=1.105)\end{array}$ \\
\hline Faixas etárias & & & & \\
20 a 29 & 5,7 & 58,0 & 25,8 & 10,5 \\
30 a 39 & 2,2 & 43,8 & 32,2 & 18,8 \\
40 a 49 & 2,9 & 38,0 & 38,6 & 20,5 \\
Total & 3,7 & 47,5 & 32,6 & 16,1 \\
\hline
\end{tabular}

Tabela 2. Distribuição de obesidade em mulheres adultas em idade reprodutiva, de acordo com fatores demográficos, socioeconômicos e reprodutivos, com correspondentes razões de prevalências. Ceará, Brasil, 2007.

\begin{tabular}{|c|c|c|c|c|}
\hline Fator de risco & $\mathrm{N}^{\circ}$ & $\begin{array}{c}\% \text { com } \\
\text { obesidade }\end{array}$ & $\begin{array}{c}\text { Razão de prevalência } \\
\text { ajustada }\end{array}$ & $\begin{array}{c}\text { Intervalo de } \\
\text { confiança a } 95 \%\end{array}$ \\
\hline \multicolumn{5}{|l|}{ Idade } \\
\hline 20 a 29 & 1.763 & 15,3 & 1,0 & \\
\hline 30 a 39 & 1.463 & 30,0 & 1,55 & $1,30-1,84$ \\
\hline 40 a 49 & 1.131 & 35,0 & 1,78 & $1,47-2,16$ \\
\hline \multicolumn{5}{|c|}{ Razão de verossimilhança ( 2 g.l.) $36,17 \quad(p<0,001)$} \\
\hline \multicolumn{5}{|l|}{ Educação } \\
\hline 12 ou mais anos & 402 & 16,9 & 1,0 & \\
\hline 9 a 11 anos & 1.616 & 19,9 & 1,17 & $0,88-1,55$ \\
\hline 5 a 8 anos & 1.158 & 28,1 & 1,33 & $1,00-1,78$ \\
\hline 0 a 4 anos & 1.038 & 32,9 & 1,40 & $1,05-1,88$ \\
\hline \multicolumn{5}{|c|}{ Razão de verossimilhança ( 3 g.l.) 8,03 ( $p=0,045)$} \\
\hline \multicolumn{5}{|l|}{ Situação conjugal } \\
\hline Solteira & 1.114 & 15,3 & 1,0 & \\
\hline Separada/viúva & 398 & 24,9 & 0,94 & $0,70-1,26$ \\
\hline Casada/em união & 2.845 & 29,4 & 1,36 & $1,11-1,67$ \\
\hline \multicolumn{5}{|c|}{ Razão de verossimilhança ( 2 g.l.) $16,74 \quad(p<0,001)$} \\
\hline \multicolumn{5}{|l|}{ Idade na menarca } \\
\hline$>12$ anos & 2.600 & 20,7 & 1,0 & \\
\hline$<12$ anos & 1.579 & 32,5 & 1,59 & $1,40-1,81$ \\
\hline \multicolumn{5}{|c|}{ Razão de verossimilhança (1 g.I.) 49,07 ( $p<0,001)$} \\
\hline \multicolumn{5}{|l|}{ Número de filhos } \\
\hline Nenhum & 1.033 & 13,2 & 1,0 & \\
\hline 1 filho & 941 & 21,1 & 1,43 & $1,11-1,86$ \\
\hline 2 ou mais & 2.383 & 32,3 & 1,65 & $1,29-2,12$ \\
\hline \multicolumn{5}{|c|}{ Razão de verossimilhança (2 g.l.) $16,46(p<0,001)$} \\
\hline \multicolumn{5}{|c|}{ Uso de anticonceptivos } \\
\hline Não & 2.197 & 18,5 & 1,0 & \\
\hline Sim & 2.160 & 32,3 & 1,31 & $1,13-1,52$ \\
\hline
\end{tabular}

a Ajustada para estas variáveis e mais: renda familiar per capita, Bolsa Família, escolaridade do chefe da família, seguro de saúde, trabalho fora de casa, número de pessoas no domicílio, história de gravidez, idade no primeiro filho e esterilização cirúrgica. 
Fatores socioeconômicos importantes como renda familiar per capita, escolaridade do chefe da família, participação no Programa Bolsa Família, trabal ho da mulher, disponibilidade de plano de saúde, que na análise univariada haviam apresentado significativas asso ciações com a obesidade, perderam a significância estatística quando incluídos nos modelos de regressão multivariada. Outros fatores igualmente importantes, como residência em zona urbana ou rural, acesso à Estratégia Saúde da Família e segurança alimentar, não apresentaram qualquer nível de associação com a obesidade.

Análise similar foi realizada somente com mulheres que apresentavam sobrepeso, comparadas às mulheres eutróficas, excluindo nestecaso aquelas que apresentavam obesidade. Este grupo de mulheres com sobrepeso se faz importante, por se encontrar num nível nutricional considerado intermediário, sendo um alvo preferencial das medidas de prevenção primária da obesidade. Observaram-se, associados ao sobrepeso, os mesmos fatores determinantes encontrados para a obesidade, com exceção do uso de métodos anticonceptivos (Tabela 3). Comparando-se os níveis de risco destas mulheres com as obesas, verificou-se que em todos os casos os riscos estimados foram inferiores, com exceção da variável nível educacional, que para as mulheres com nível primário apresentou um risco maior de sobrepeso (49\%) em comparação ao verificado para obesidade (40\%).

Tabela 3. Distribuição de sobrepeso em mulheres adultas em idade reprodutiva, de acordo com fatores demográficos, socioeconômicos e reprodutivos, com correspondentes razões de prevalências. Ceará, Brasil, 2007.

\begin{tabular}{|c|c|c|c|c|}
\hline Fator de risco & $\mathrm{N}^{\circ}$ & $\begin{array}{c}\% \text { com } \\
\text { sobrepeso }\end{array}$ & $\begin{array}{c}\text { Razão de prevalência } \\
\text { ajustada }\end{array}$ & $\begin{array}{c}\text { Intervalo de } \\
\text { confiança a } 95 \%\end{array}$ \\
\hline \multicolumn{5}{|l|}{ Idade } \\
\hline 20 a 29 & 2.158 & 30,8 & 1,0 & \\
\hline 30 a 39 & 1.846 & 44,5 & 1,33 & $1,18-1,49$ \\
\hline 40 a 49 & 1.482 & 50,4 & 1,56 & $1,38-1,77$ \\
\hline \multicolumn{5}{|c|}{ Razão de verossimilhança (2 g.I.) 48,92 ( $p<0,001)$} \\
\hline \multicolumn{5}{|l|}{ Educação } \\
\hline 12 ou mais anos & 474 & 29,5 & 1,0 & \\
\hline 9 a 11 anos & 2.009 & 35,6 & 1,32 & $1,08-1,63$ \\
\hline 5 a 8 anos & 1.508 & 44,8 & 1,46 & $1,19-1,80$ \\
\hline 0 a 4 anos & 1.311 & 46,8 & 1,49 & $1,20-1,84$ \\
\hline \multicolumn{5}{|c|}{ Razão de verossimilhança ( 3 g.l.) 15,89 ( $p=0,001)$} \\
\hline \multicolumn{5}{|l|}{ Situação conjugal } \\
\hline Solteira & 1.365 & 30,8 & 1,0 & \\
\hline Separada/viúva & 471 & 36,5 & 0,86 & $0,70-1,06$ \\
\hline Casada/em união & 3.648 & 44,9 & 1,19 & $1,04-1,36$ \\
\hline \multicolumn{5}{|c|}{ Razão de verossimilhança ( 2 g.l.) 18,09 ( $p<0,001)$} \\
\hline \multicolumn{5}{|l|}{ Idade na menarca } \\
\hline$>12$ anos & 3.391 & 39,2 & 1,0 & \\
\hline$<12$ anos & 1.863 & 42,8 & 1,15 & $1,05-1,27$ \\
\hline \multicolumn{5}{|c|}{ Razão de verossimilhança ( 1 g.l.) 8,86 ( $p=0,003)$} \\
\hline \multicolumn{5}{|l|}{ Número de filhos } \\
\hline Nenhum & 1.255 & 28,5 & 1,0 & \\
\hline 1 filho & 1.179 & 37,1 & 1,22 & $1,04-1,43$ \\
\hline 2 ou mais & 3.052 & 47,1 & 1,32 & $1,13-1,53$ \\
\hline
\end{tabular}

a Ajustada para estas variáveis e mais: renda familiar per capita, Bolsa Família, escolaridade do chefe da família, seguro de saúde, trabalho fora de casa, número de pessoas no domicílio, história de gravidez, idade no primeiro filho, uso de métodos anticonceptivos e esterilização cirúrgica. 
A Tabela 4 apresenta a análise ajustada da associação entre características de morbidade e nutricionais ea obesidade e o sobrepeso. Mulheres com obesidade excessiva apresentaram um risco 3,1 vezes maior deterem recebido diagnóstico médico de hipertensão arterial, em comparação com as mulheres eutróficas, enquanto as com sobrepeso apresentaram $54 \%$ de excesso de risco de terem sido diagnosticadas como hipertensas. Em relação ao diabetes mellitus, o excesso de obesidade faz o risco desta morbidade dobrar, em relação às mulheres sem distúrbios nutricionais.

Conforme seria de se esperar, quatro em cada cinco mulheres com obesidade não estavam satisfeitas com a sua imagem corporal, o mesmo acontecendo com a metade das mulheres com sobrepeso. Em termos derisco, independentemente do grau de obesidade, mulheres com a sua con- dição apresentavam 4,3 vezes maior probabilidade de insatisfação com o corpo em relação àquelasqueapresentavam eutrofia. Esteexcesso derisco $(2,9)$ também foi apresentado pelas mulheres que estavam com peso acima do recomendado, mas ainda fora da faixa de obesidade.

M enos de um terço das mulheres obesas estavam procurando corrigir o distúrbio realizando ações que as levassem à perda de peso, o mesmo acontecendo com uma em cada cinco muIheres com sobrepeso. M esmo com estas relativamente baixas proporções, a probabilidade de as mulheres obesas se engajarem em algum programa deperda de peso foi 2,7 vezes mais el evada do que as mulheres com IMC na faixa considerada normal. Assim como no caso da insatisfação corporal, um grau de obesidade mais elevado não implicou maior probabilidade de se adotar a atitude de perder peso.

Tabela 4. Características de saúde e nutricionais de mulheres adultas em idade reprodutiva, de acordo com 0 estado nutricional, com as correspondentes razões de prevalência. Ceará, Brasil, 2007.

\begin{tabular}{|c|c|c|c|c|}
\hline Fator de risco & $\operatorname{Sim}(\%)$ & Não (\%) & $\begin{array}{c}\text { Razão de prevalência } \\
\text { ajustadaa }\end{array}$ & $\begin{array}{c}\text { Intervalo de } \\
\text { confiança a } 95 \%\end{array}$ \\
\hline \multicolumn{5}{|l|}{ Hipertensão arterial } \\
\hline Eutrofia & $225(7,3)$ & $2.847(92,7)$ & 1,00 & \\
\hline Sobrepeso & $311(14,8)$ & $1.791(85,2)$ & 1,54 & $1,28-1,86$ \\
\hline Obesidade, grau I & $172(22,2)$ & $603(77,8)$ & 2,11 & $1,69-2,63$ \\
\hline Obesidade, grau II & $86(32,1)$ & $182(67,9)$ & 3,11 & $2,35-4,11$ \\
\hline \multicolumn{5}{|c|}{ Razão de verossimilhança ( 3 g.l.) 80,92 ( $p<0,001)$} \\
\hline \multicolumn{5}{|l|}{ Diabetes mellitus } \\
\hline Eutrofia & $112(3,5) 119$ & $3.045(96,5)$ & 1,00 & \\
\hline Sobrepeso & $(5,4)$ & $2.065(94,6)$ & 1,31 & $0,99-1,74$ \\
\hline Obesidade, grau I & $48(5,9)$ & $761(94,1)$ & 1,43 & $1,00-2,05$ \\
\hline Obesidade, grau II & $26(9,3)$ & $255(90,7)$ & 2,08 & $1,29-3,34$ \\
\hline \multicolumn{5}{|c|}{ Razão de verossimilhança ( 3 g.l.) 11,09 ( $p=0,011)$} \\
\hline \multicolumn{5}{|l|}{ Satisfação com o corpo } \\
\hline Eutrofia & $544(19,9)$ & $2.186(80,1)$ & 1,00 & \\
\hline Sobrepeso & $1.168(55,3)$ & $944(44,7)$ & 2,86 & $2,57-3,19$ \\
\hline Obesidade, grau I & $645(80,2)$ & $159(19,8)$ & 4,09 & $3,61-4,62$ \\
\hline Obesidade, grau II & $229(83,6)$ & $45(16,4)$ & 4,26 & $3,61-5,04$ \\
\hline \multicolumn{5}{|c|}{ Razão de verossimilhança ( 3 g.l.) 590,07 ( $p<0,001)$} \\
\hline \multicolumn{5}{|c|}{ Ações de perda de peso } \\
\hline Eutrofia & $345(11,3)$ & $2.697(88,7)$ & 1,00 & \\
\hline Sobrepeso & $424(19,9)$ & $1.708(80,1)$ & 1,82 & $1,56-2,12$ \\
\hline O besidade, grau I & $238(29,6)$ & $566(70,4)$ & 2,75 & $2,29-3,29$ \\
\hline Obesidade, grau II & $82(29,4)$ & $197(70,6)$ & 2,73 & $2,10-3,57$ \\
\hline \multicolumn{5}{|c|}{ Razão de verossimilhança ( 3 g.l.) 140,74 ( $p<0,001)$} \\
\hline
\end{tabular}




\section{Discussão}

A faixa etária reprodutiva investigada no presente estudo inclui mulheres no início da idadeadultae em sua plena maturidade, correspondendo também à faixa de maior desempenho produtivo da mulher. Considerando a importância do grupo populacional, a constatação de quea metade dessas mulheres já apresenta distúrbios nutricionais em graus variados é motivo de preocupação. Observou-se que o problema é de início precoce, uma vez que já a partir de uma faixa tão jovem como os 30 anos cerca de $20 \%$ das mulheres apresentam IM C compatível com a obesidade. Resultados similares foram encontrados em estudo de base populacional realizado na região Sul com mulheres de 20 a 60 anos, o qual encontrou prevalências de $32 \%$ de sobrepeso e de $18 \%$ de obesidade $^{18}$. Em contraste, estudo realizado com mulheres residentes nas capitais dos 27 estados brasileiros observou na faixa etária de 25 a 54 anos, também, mais da metade das mulheres com excesso de peso, mas com uma possível sobre estimativa de sobrepeso, alcançou cerca de $40 \%$, e uma subestimativa de obesidade, em torno de $10 \%{ }^{27}$. As diferenças entre os dois estudos incluíram a não representação da população rural e 0 registro do peso e altura autorreferidos no estudo nacional. Estes resultados sinalizam para o início da promoção de ações de prevenção da obesidade já na terceira década de vida, tendo como foco principalmenteo enorme contingente de mulheres que se encontram na condição de sobrepeso.

Somadas à idade, a escolaridade e a situação conjugal se caracterizaram como os principais fatores sociodemográficos determinantes da obesidade e do sobrepeso. Observou-se um significativo gradiente de risco para o nível de escolaridade decrescente, com mulheres que reportaram menos de cinco anos de estudos apresentando uma probabilidade $40 \%$ maior de serem obesas em comparação a mulheres com nível superior. Estudos realizados com a população feminina do país, e especificamente da região N ordeste, encontraram a mesma associação inversa entre nível deescolaridadee os desfechos nutricionais ${ }^{27,28}$. Esta constatação éimportante, pois impõe que as medidas educativas voltadas para a redução do problema sejam especialmentesensíveis às pessoas de limitado nível de compreensão.

A condição de solteira, separada ou viúva, ou seja, de não estar engajada em uma relação mais estável, parece proteger a mulher dos distúrbios nutricionais, em comparação às mulheres casa- das ou em união conjugal, que apresentaram um risco 36\% maior deserem obesas. É provável que neste caso o excesso de risco esteja relacionado a uma maior preocupação com a imagem corporal e a uma vida social mais ativa por parte daquelas que não vivem com um parceiro, e a uma menor dedicação das mulheres em união a si próprias, por conta dos esforços que despendem com os cuidados do lar e dos filhos. Esta relação entreunião estável ea ocorrência de obesidadejá foi registrada em outros estudos realizados com a população brasileir $a^{27}$ e american $a^{29}$. N este estudo longitudinal realizado nos EUA, observouse que mulheres solteiras no início do acompanhamento equesecasaram posteriormenteapresentaram maior ganho de peso do que mulheres que estavam casadas em ambos os momentos do seguimento.

Os fatores relacionados à função reprodutiva da mulher receberam um enfoque especial neste estudo e, com efeito, apresentaram-se como importantes determinantes para o so brepeso e, principalmente, a obesidade. A idade quando do primeiro episódio menstrual, o números de filhos e o uso de métodos anticonceptivos foram os fatores reprodutivos que se destacaram significativamente, em meio aos diversos outros analisados. U ma menarca em idade precoce, ou seja, antes dos 12 anos, aumentou em $59 \%$ o risco de obesidade. A menarcaéum fenômeno tardio na evolução puberal, estando a sua ocorrência sujeita a vários fatore ${ }^{30}$, destacando-se entre el es 0 estado nutricional e o padrão de crescimento durante a infância ${ }^{31}$. 0 período pós-menarca, por seu turno, secaracteriza pela diminuição do incremento da estatura epelo característico aumento depeso ${ }^{30}$. A maioria dos estudos realizados no Brasil, que exploraram a relação entre a menarca e o estado nutricional, limitaram-se à investigação de seus efeitos duranteo período da adolescência. Estudo realizado com mulheres adultas na região Sul do Brasil observou um similar efeito protetor da menarca tardia contra a obesidade ${ }^{18}$. Esta associação é preocupante, pois a puberdade tem apresentado uma tendência de ocorrer cada vez mais cedo, estimando-se que a cada década a idade da menarca diminua de três a quatro meses ${ }^{32}$, implicando assim a manutenção e o agravamento deste fator de risco de natureza biológica.

0 nascimento do primeiro filho já aumenta em $43 \%$ o risco de obesidade na mulher. Com o segundo filho, este risco cresce para $65 \%$, não aumentando mais significativamente com a elevação do número de filhos. Em estudo realizado com mulheres da região Sul, a paridade também 
foi identificada como associada à obesidade, mas com um excesso de risco cinco vezes maior para as multíparas com mais de quatro filhos ${ }^{18}$. Esteé novamente um achado preocupante, pois dois filhos éa quantidade correntemente considerada ideal de crianças por parte dos casais. Estudos prévios mostram quea gestação égeralmenteum período de risco para ganho de peso, que tendea não ser perdido após o parto, fato este que deve se agravar ainda mais quando há um reduzido espaçamento em relação a uma gravidez anterior ${ }^{33-36}$. Mulheres em idade reprodutiva deveriam, portanto, figurar permanentemente no foco de programas de intervenção e educação nutricional, recebendo orientações adequadas quanto à prevenção do peso excessivo na gravidez e sobre os meios de retornarem ao peso pré gestacional ${ }^{35-37}$.

Cerca de $55 \%$ das mulheres utilizam algum método para evitar filhos; destas, $51 \%$ fazendo uso do anticonceptivo oral (dados não mostrados). 0 uso de métodos anticonceptivos aumentou em $31 \%$ o risco de obesidade, não se mostrando significativamente associado ao sobrepeso. 0 desenho transversal do presenteestudo não permite elucidar qual dos dois fatores é efeito constatando-se, todavia, que eles coexistem. No entanto, preocupa saber que o risco de tromboembolismo venoso aumenta com a obesidade, e que esterisco pode ser aditivo quando usado um método hormonal combinado ${ }^{38}$.

Duas formas demorbidade de alta relevância em saúde pública, a hipertensão arterial e o diabetes mellitus, foram analisadas neste estudo quanto à sua associação com a obesidade e 0 sobrepeso. Os distúrbios nutricionais estiveram significativamente associados a ambos os desfechos, apresentando gradientes de risco importantes, que reforçam a evidência de uma relação causal. Deste modo, mulheres com um grau maior de obesidade (IM C>=35,0) apresentaram probabilidade duas e três vezes maiores de reportarem terem sido diagnosticadas com diabe tes ehipertensão, respectivamente, em comparação a mulheres eutróficas, mesmo após estas associações serem controladas para uma série de variáveis socioeconômicas, demográficas e de saúde. Diversos estudos têm demonstrado a associação entrea obesidadee doen ças crônicas em mulheres, como a hipertensão 7,10,27,39,40-42 e o diabetes $7,10,27,39,42$, inclusiveno que diz respeito às suas formas mais graves, que requerem hospitalização ${ }^{11}$, sempre enfatizando a importância da prevenção edo tratamento da obesidade para o controle destas enfermidades.
Desfechos de natureza comportamental relacionados à obesidade foram também analisados, observando-se que mais de $80 \%$ das mulheres obesas, em qualquer grau, mostravam-se insati ffeitas com a sua imagem corporal. Esse fato ocorreu com uma probabilidade quatro vezes maior em relação às mulheres sem distúrbios nutricionais. A pesar do elevado índice de insatisfação, entretanto, somente $30 \%$ das mulheres com obesidade tomavam alguma ação para reduzir o peso, tais como dietas, atividades físicas ou uso de medicamentos. Diversos estudos têm encontrado resultados semelhantes ${ }^{14-17,43}$, sendo importanteressaltar que os casos, frequentes, que fracassam nas tentativas de emagrecimento tendem a sofrer uma baixa na autoestima, resultando no desprezo pelas ações que visam ao controle do peso ${ }^{44}$.

Embora estudos mostrem associação entre fatores econômicos e distúrbios nutricionai s $^{21,28}$, neste as variáveis de natureza econômica, como a renda familiar, a segurança alimentar representada pelo Programa Bolsa Família, a escolaridade do chefe da família, o trabal ho da mulher ea residência em zona urbana/rural, não se consolidaram como fatores determinantes da obesidade e do sobrepeso, após serem controladas para os demais fatores, o que também ocorreu com as variáveis relacionadas à saúde, como a disponibilidade de seguro e acesso à Estratégia Saúde da Família.

Conclui-se que características reprodutivas são importantes fatores determinantes da obesidade e do sobrepeso na população de mulheres adultas em idade fértil, na região semiárida do Brasil. Constata-se ainda que estes fatores (menarca precoce, número de filhos e contracepção) são praticamente não redutíveis, sendo importantes, entretanto, para demarcarem grupos de maior risco, sobre os quais as mensagens voltadas para prevenção e controle do sobrepeso/obesidade devem prioritariamenteincidir. Nestesentido, recomenda-se o envolvimento ativo dos programas/serviços de planejamento familiar e pré-natal no combate à obesidade, admitindose que uma proporção considerável de mulheres com os problemas mencionados concentra-se entre suas usuárias.

Considerando queo nível educacional incipiente foi um importante fator de risco detectado, esendo este passível de intervenção, recomendase, a médio e longo prazos, maior acesso das mulheres à escola, com uma consequente el evação do seu nível de escolarização. A curto prazo, recomenda-se maior preocupação em traduzir 
as mensagens educativas sobre alimentação e nutrição para uma linguagem acessível às pessoas com pouca instrução formal, transmitindo de forma simples, clara e objetiva os meios pelo quais, por exemplo, as dietas inadequadas levam ao acúmulo de gordura corporal, e por conse guinte à obesidade.

Reduzir a prevalência da obesidade e do sobrepeso seguramente implica ganhos importantes em termos de saúde física e mental, como o controle de graves enfermidades crônicas e 0 aumento do sentimento de autoestima na população, devendo-se, para isto, investir-se maciçamente em medidas de prevenção tanto primária (redução do sobrepeso) como secundária (redução da obesidade). Vale a pena ressaltar, por fim, que o presente estudo focalizou uma faixa demulheres relativamentejovens, sabendo-seque o problema do sobrepeso/obesidade agrava-se tanto em termos de magnitude como em consequências mais severas, nas faixas etárias mais idosas. É, entretanto, nas camadas mais jovens da população que as medidas voltadas às mudanças de comportamento podem propiciar mai or efeito, sendo, assim, a faixa etária enfocada neste estudo a mais efetiva para a concentração de esforços voltados ao combate ao sobrepeso e à obesidade.

\section{Colaboradores}

LL Correia, DMI Silveira, AC Silva, JS Campos, M LM Bosi, M MT M achado, HAL Rocha, AJLA Cunha e AC Lindsay participaram das etapas de redação, revisão e aprovação da versão final do artigo.

\section{Agradecimentos}

Ao Consel ho Nacional de Desenvolvimento $\mathrm{Ci}$ entífico e Tecnológico (CN Pq), que, através do Edital M CT-CN Pq/M S-DAB/SAS, proporcionou 0 apoio técnico-financeiro indispensável ao desenvolvimento do presenteestudo. Agradecemos igualmente à Escola de Saúde Pública do Ceará, às prefeituras dos municípios envolvidos e aos entrevistadores por todo o empenho em viabilizar a realização da pesquisa. 
1. Mokdad AH, Serdula MK, Dietz WH, Bowman BA, $M$ arks JS, Koplan JP. The spread of the obesity epidemic in the United States, 1991-1998. JAM A 1999; 282:1519-1522.

2. Shafique $S$, Akhter N, Stallkamp G, de Pee $S, P a-$ nagides $D$, Bloem $M W$. Trends of under-and overweight among rural and urban poor women indicate the double burden of malnutrition in Bangladesh. Int J Epidemiol 2007; 36(2):449-457.

3. Fine JT, Colditz GA, Coakley EH, Moseley G, Manson JE, Willett WC, Kawachi I. A prospective study of weight change and health-related quality of life in women. JAM A 1999; 282:2136-2142.

4. Ke-You G, Da-Wei F. The magnitude and trends of under- and over-nutrition in Asian countries. Biomed Environ Sci 2001; 14(1-2):53-60.

5. Caballero B. Symposium: obesity in developing countries: biological and ecological factors. J Nutr 2001; 131(3):866S-870S.

6. Puoane T, Steyn K, Bradshaw D, Laubscher R, Fourie J, Lambert V, M bananga N. O besity in South Africa: the South African Demographic and Health Survey. Obesity Research 2002; 10(10):1038-1048.

7. World Health Organization. O besity: preventing and managing the global epidemic: Technical Report of a WHO Expert Consultation on O besity, 894:1253, Geneva: WHO; 2000.

8. Ogden $C L$, Carroll MD, Curtin LR, MCD owell MA, Tabak CJ, Flegal KM. Prevalence of overweight and obesity in the United States, 1999-2004. JAM A 2006; 295:1549-1555

9. Caterson ID, Gill TP. Obesity: epidemiology and possible prevention. Best Pract Res Clin Endocrinol M etab 2002; 16:595-610.

10. Cercato C, M ancini MC, Arguello AMC, Passos VQ, Villares SM F, Halpern A. Systemic hypertension, diabetes mellitus, and dislipidemia in relation to body mass index: evaluation of a Brazilian population. Rev Hosp Clín Fac Med S Paulo 2004; 59(3):113-118.

11. Sichieri R, Nascimento S, Coutinho W. Importância e custo das hospitalizações associadas ao sobrepeso e obesidade no Brasil. Cad Saude Publica 2007; 23(7):1721-1727.

12. World Health Organization. Diet, nutrition and the prevention of chronic diseases: report of a WHO Study Group. Geneva: World Health Organization; 1990. (WHO Technical Report Series, n. 797).

13. York DA, Rössner $S$, Caterson I, Chen CM, Jame WP, Kumanyika S, M artorell R, Vorster HH. Prevention Conference VII: O besity, a worldwide epidemic related to heart disease and stroke: Group I: Worldwide demographics of obesity. Circulation 2004; 110(18):e463-470.

14. Schmalz DL. "I feel fat": weight-related stigma, body esteem, and BMI as predictors of perceived competence in physical activity. O bes Facts 2010; 3(1):15-21.
15. Palmeira $A L, M$ arkland $D A$, Silva $M N$, Branco $T L$, M artins SC, M inderico CS, Vieira PN, Barata JT, Serpa SO, Sardinha LB, Teixeira PJ. Reciprocal effects among changes in weight, body image, and other psychological factors during behavioral obesity treatment: a mediation analysis. Int J Behav Nutr Phys Act 2009; 6:9.

16. Riley NM, Bild DE, Cooper L, Schreiner $P$, Smith $D E$, Sorlie $P$, Thompson JK. Relation of self-image to body size and weight loss attempts in black women: the Cardia study: Coronary Artery Risk Development in Young Adults. Am J Epidemiol 1998; 148(11):1062-1068.

17. Lynch E, Liu K, Wei GS, Spring B, Kiefe C, Greenland $P$. The relation between body size perception and change in body mass index over 13 years: the Coronary Artery Risk Development in Young Adults (Cardia) study. Am J Epidemiol 2009; 169(7):857-866.

18. Teichmann L, Olinto M TA, Costa JSD, Ziegler D. Fatores de risco associados ao sobrepeso e à obesidade em mulheres de São Leopoldo, RS. Rev Bras Epidemiol 2006; 9(3):360-373.

19. Florêncio TM, Ferreira HS, França AP, Cavalcante JC, Sawaya AL. Obesity and undernutrition in a very-low-income population in the city of Maceió, Northeastern Brazil. Br J Nutr 2001; 86(2):277-284.

20. Lombard C, Deeks A, Jolley D, Teede HJ. Preventing weight gain: the baseline weight related behaviors and delivery of a randomized controlled intervention in community based women. BM C Public Health 2009; 9:2.

21. Monteiro CA, Moura EC, Conde WL, Popkin BM . Socioeconomic status and obesity in adult populations of developing countries: a review. Bull World Health Organ 2004; 82(12):940-946.

22. Barros FC, Victora CG. Epidemiologia da saúde infantil: um manual para diagnósticos comunitários. São Paulo: Hucitec/Unicef; 1991.

23. Coutinho LMS, Scazufca $M, M$ enezes $P R$. M étodos para estimar razão de prevalência em estudos de corte transversal. Rev Saude Publica 2008; 42(6):992998.

24. M isago C, Fonseca W, Correia LL, Femandes LM F, Campbell O. Determinants of abortion among women admitted to hospitals in Fortaleza, Northeastern Brazil. Int J Epidemiol 1998; 27:833-839.

25. Zoccheti C, Consonni D, Bertazzi PA. Relationship between prevalence rate ratios and odds ratios in cross-sectional studies. Int J Epidemiol 1997; 26:220223.

26. Victora CG, Hutty SR, Fuchs SC, Olinto MTA. The role of conceptual frameworks in epidemiological analysis: a hierarchical approach. Int J Epidemiol 1997; 26:224-227.

27. Gigante DP, Moura EC, Sardinha LM V. Prevalência de excesso de peso e obesidade e fatores associados, Brasil, 2006. Rev Saude Publica 2009; 43(Supl.2): 83-89. 
28. M onteiro CA, Conde WL, Popkin BM . Independent effects of income and education on the risk of obesity in the Brazilian adult population. J Nutr 2001; 131(3):881-886.

29. Sobal J, Rauschenbach B, Frongillo EA. M arital status changes and body weight changes: a US Iongitudinal analysis. Soc Sci M ed 2003; 56(7):1543-1555.

30. Vitalle MSS, Tomioka CY, Juliano Y, Amancio OM S. índice de massa corporal, desenvolvimento puberal e sua relação com a menarca. Rev Assoc Med Bras 2003; 49(4):429-433.

31. Martínez J, Araújo C, H orta, BL, Gigante DP. Growth patterns in early childhood and the onset of menarche before age twelve. Rev Saude Publica 2010; 44(2):249-260.

32. Marshall WA, Tanner SM. Variations in pattern of puberal chances in girls. Arch Dis Child 1969; 44:291303.

33. Fraser $A$, Tilling $K$, MacDonald-Wallis $C$, Sattar $N$, Brion MJ, Benfield L, N ess A, Deanfield J, Hingorani A, Nelson SM, Smith GD, Lawlor DA. Association of maternal weight gain in pregnancy with offspring obesity and metabolic and vascular traits in childhood. Circulation 2010; 121(23):2557-2564.

34. Brown K, Apuzzio J, Weiss G. Maternal obesity and associated reproductive consequences. Womens Health 2010; 6(2):197-203.

35. Weisman CS, Hillemeier M M, Downs DS, Chuang $\mathrm{CH}$, Dyer AM. Preconception predictors of weight gain during pregnancy: prospective findings from the Central Pennsylvania Women's Health Study. Womens Health Issues 2010; 20(2):126-132.

36. Mottola M F, Giroux I, Gratton R, Hammond JA, $\mathrm{H}$ anley $\mathrm{A}, \mathrm{H}$ arris $\mathrm{S}, \mathrm{MCM}$ anus $\mathrm{R}$, Davenport $\mathrm{MH}$, Sopper M M. Nutrition and exercise prevent excess weight gain in overweight pregnant women. M ed Sci Sports Exerc 2010; 42(2):265-272.

37. Peterson KE, Sorensen $G$, Pearson $M$, Hebert JR, Gottlieb BR, M cCormick MC. Design of an intervention addressing multiple levels of influence on dietary and activity patterns of low-income, postpartum women. Health Educ Res 2002; 17(5):531-540.

38. M urthy AS. Obesity and contraception: emerging issues. Semin Reprod Med 2010; 28(2):156-163.

39. Martins IS, Oliveira DC, Marinho SP, Araújo EAC. Hipertensão em segmentos sociais pauperizados da região do Vale do Paraíba - São Paulo. Cien Saude Colet 2008; 13(2):477-486.

40. Borges HP, Cruz NC, Moura EC. Associação entre hipertensão arterial e excesso de peso em adultos, Belém, Pará, 2005. Arq Bras Cardiol 2008; 91(2):110118.

41. Pitanga FJG, Lessa I. Associação entre indicadores antropométricos de obesidade e risco coronariano em adultos na cidade de Salvador, Bahia, Brasil. Rev Bras Epidemiol 2007; 10(2):239-248.
42. Souza LJ, Neto CG, Chalita FEB, Reis AFF, Bastos DA, Filho JTDS, Souza TF, Côrtes VA. Prevalência de obesidade e fatores de risco cardiovascular em Campos, Rio de Janeiro. Arq Bras Endocrinol M etab 2003; 47(6):69-74.

43. Andrade A, Bosi M LM. Mídia e subjetividade: impacto no comportamento alimentar feminino. Rev Nutr [periódico na Internet]. 2003 jan [citado 2010 jul 12]; 16(1):117-125. Disponível em: http://www. scielo.br/scielo.php?script=sci_arttext $\&$ pid $=$ S1415-52732003000100012\&lng=pt

44. Fett CA, Fett WCR, Marchini JS, Ribeiro RPP. Estilo de vida e fatores de risco associados ao aumento da gordura corporal de mulheres. Cien Saude Colet 2010; 15(1):131-140.

Artigo apresentado em 10/03/2010

A provado em 05/07/2010

Versão final apresentada em 05/08/2010 